\title{
High-resolution bacterial 16S rRNA gene profile meta-analysis and biofilm status reveal common colorectal cancer consortia
}

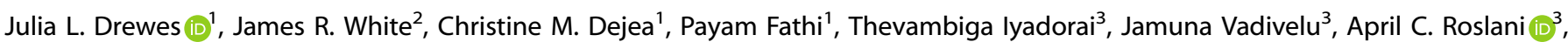
Elizabeth C. Wick ${ }^{1}$, Emmanuel F. Mongodin ${ }^{4}$, Mun Fai Loke ${ }^{3}$, Kumar Thulasi ${ }^{3}$, Han Ming Gan ${ }^{5}$, Khean Lee Goh ${ }^{3}$, Hoong Yin Chong ${ }^{3}$, Sandip Kumar ${ }^{3}$, Jane W. Wanyiri ${ }^{1}$ and Cynthia L. Sears ${ }^{1}$

Colorectal cancer (CRC) remains the third most common cancer worldwide, with a growing incidence among young adults. Multiple studies have presented associations between the gut microbiome and CRC, suggesting a link with cancer risk. Although CRC microbiome studies continue to profile larger patient cohorts with increasingly economical and rapid DNA sequencing platforms, few common associations with CRC have been identified, in part due to limitations in taxonomic resolution and differences in analysis methodologies. Complementing these taxonomic studies is the newly recognized phenomenon that bacterial organization into biofilm structures in the mucus layer of the gut is a consistent feature of right-sided (proximal), but not left-sided (distal) colorectal cancer. In the present study, we performed 16S rRNA gene amplicon sequencing and biofilm quantification in a new cohort of patients from Malaysia, followed by a meta-analysis of eleven additional publicly available data sets on stool and tissuebased CRC microbiota using Resphera Insight, a high-resolution analytical tool for species-level characterization. Results from the Malaysian cohort and the expanded meta-analysis confirm that CRC tissues are enriched for invasive biofilms (particularly on rightsided tumors), a symbiont with capacity for tumorigenesis (Bacteroides fragilis), and oral pathogens including Fusobacterium nucleatum, Parvimonas micra, and Peptostreptococcus stomatis. Considered in aggregate, species from the Human Oral Microbiome Database are highly enriched in CRC. Although no detected microbial feature was universally present, their substantial overlap and combined prevalence supports a role for the gut microbiota in a significant percentage (>80\%) of CRC cases.

npj Biofilms and Microbiomes (2017)3:34; doi:10.1038/s41522-017-0040-3

\section{INTRODUCTION}

While numerous environmental risk factors for colorectal cancer (CRC) were established decades ago, ${ }^{1}$ only in recent years have we begun to appreciate the role of the gut microbiome in CRC. Over a dozen studies have now established that CRC is associated with some form of microbial dysbiosis in terms of taxonomic composition and/or mucosal structural organization (e.g., biofilms), as detected either via stool or tumor tissue. ${ }^{2-16}$ However, these CRC microbiome profiling studies have yielded inconsistent findings, likely due to differences in study design and analysis methodologies. Furthermore, the relative contribution of mucosal vs. luminal (i.e., stool) populations, known to differ, ${ }^{17}$ is uncertain. In the present study, we analyzed the taxonomic composition and biofilm status of both previous and new cohorts in order to present a more robust depiction of the contribution of the microbiota to CRC.

\section{RESULTS}

Confirmation of the biofilm-spatial relationship in CRC

We first sought to validate previous findings that invasive biofilms are a feature of right-sided (proximal) $C R C$, originally described in a USA cohort (USA, N=36) and a Malaysian cohort (MAL1,
$N=22) .^{16}$ Biofilms form when bacteria aggregate on a surface (biological or inert) and become encased in a polymeric matrix. $^{18,19}$ Similar to other in vivo biofilms including oral biofilms (dental plaque) and Pseudomonas aeruginosa biofilms in cystic fibrosis patients, the colonic biofilm matrix may consist of both host mucus and bacteria-produced extrapolymeric substrates. ${ }^{20,21}$ The invasion and attachment of biofilms to the colonic epithelium and inner mucus layer can be visualized by a number of different microscopy-based methodologies including fluorescence in situ hybridization (FISH) staining with oligonucleotide fluorescent probes, electron microscopy, or acridine orange staining. ${ }^{22,23}$ Tumor and paired normal samples from a new cohort of 23 patients in Malaysia who underwent surgical resection (described hereafter as MAL2; see Supplementary Table S1 for metadata) were thus screened by FISH with the Eub338 universal 16S rRNA gene probe, using slides from tissue blocks fixed in Carnoy's to preserve the mucus layer. Paired normal samples were taken from a non-cancerous region of the resected colorectal sample as far as possible from the tumor itself (Supplementary Fig. S1a). Representative biofilm-positive (right-sided) and biofilm-negative (left-sided) tissues from MAL2 are shown in Fig. 1a. A total of 6/16 left-sided tumors from MAL2 were biofilm positive (37.5\%), while all seven right-sided tumors were biofilm positive (100\%). Biofilm

\footnotetext{
'Johns Hopkins University School of Medicine, 1550 Orleans St., Baltimore, MD 21231, USA; ${ }^{2}$ Resphera Biosciences, 1529 Lancaster St., Baltimore, MD 21231 , USA; ${ }^{3}$ University of

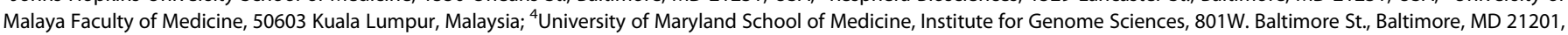
USA and ${ }^{5}$ Monash University Malaysia, School of Science, 47500 Bandar Sunway, Selangor, Darul Ehsan, Malaysia

Correspondence: Cynthia L. Sears (csears@jhmi.edu)

Julia L. Drewes and James R. White contributed equally to this work.
}

Received: 6 June 2017 Revised: 12 October 2017 Accepted: 16 October 2017

Published online: 29 November 2017 


\section{A}
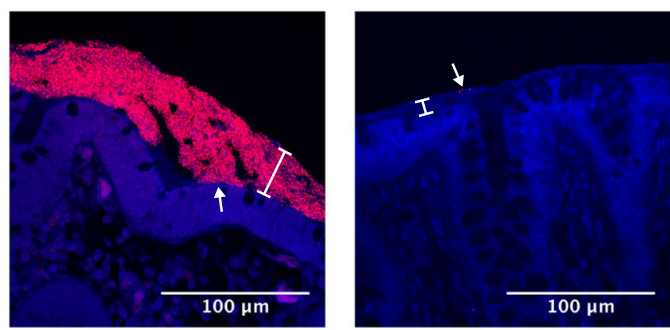

C

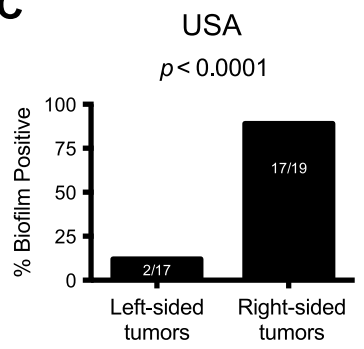

D

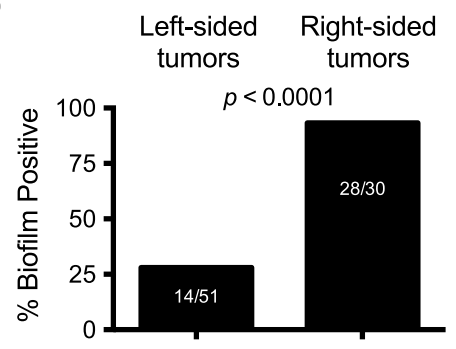

All Tumors

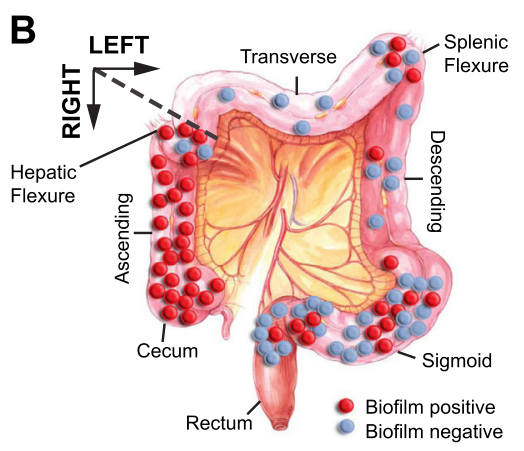

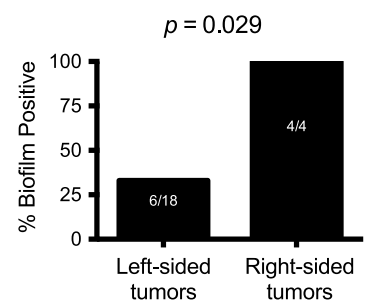

MAL2

$p=0.008$

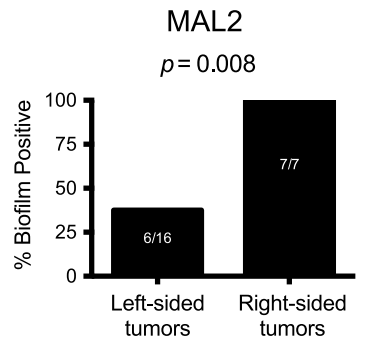

E

Left-sided tumors

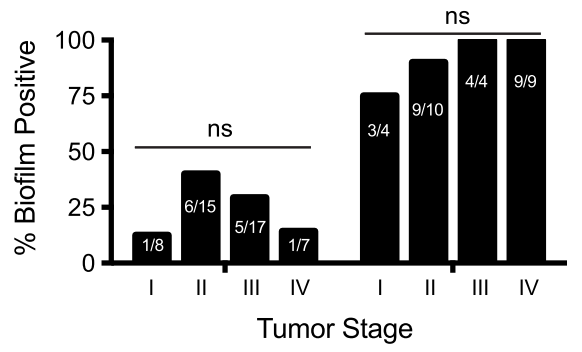

F
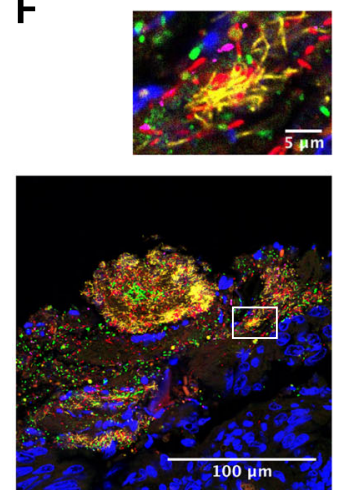
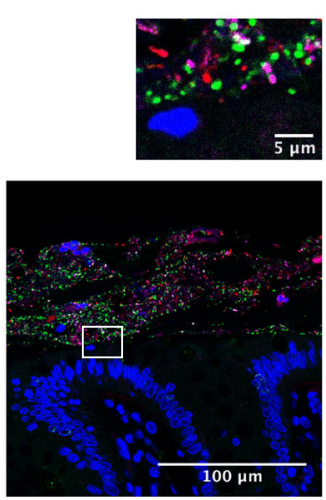

G
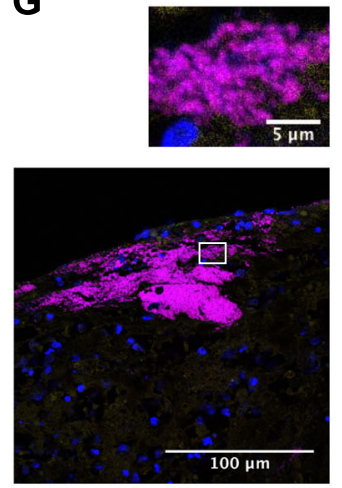
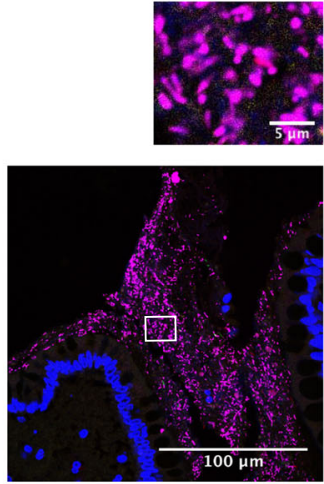

Fig. 1 Meta-analysis of biofilm prevalence in CRC. a Microbial biofilms in Carnoy's-fixed tissue were detected by FISH with the universal 16S rRNA gene probe Eub338 (stained in red) and the nucleic acid stain DAPI (blue). White brackets denote the mucus layer, visible via autofluorescence of the tissue. Left panel: a biofilm-positive paired normal tissue from the right colon of a CRC patient with abundant bacteria adjacent to the epithelium (arrow). Right panel: a biofilm-negative paired normal tissue from the left colon of a CRC patient with minimal bacteria (arrow) present only in the outer edge of the mucus. Scale bars represent $100 \mu \mathrm{m}$. b The locations of tumors from USA, MAL1, and MAL2 are overlaid on a diagram of the colon. c Percentage of biofilm-positive right and left tumors for the three cohorts. All statistics shown are Fisher's exact tests. d Total percentage of biofilm-positive right- and left-sided tumors from all three cohorts is depicted with a Fisher's exact test $p$-value. e Left-sided and right-sided tumors separated according to tumor stage and biofilm status. Multivariate logistic regression analysis was performed after controlling for tumor side. f, $\mathbf{g}$ Biofilm-positive samples were stained with DAPI (blue) and probes against four bacterial membership groups: Fusobacterium (yellow), Bacteroidetes (green), Lachnospiraceae (red), and Proteobacteria (magenta). Scale bars in large images represent $100 \mu \mathrm{m}$; scale bars in smaller, inset images represent $5 \mu \mathrm{m}$. $\mathbf{f}$ Representative polymicrobial biofilm from a tumor (left panel) and its paired normal tissue (right panel), with blooms of Fusobacterium in yellow visible only in the tumor. g Representative Proteobacteria-dominant tumor (left panel) and its paired normal tissue (right panel) 
results for MAL2 were then combined with the previously published USA and MAL1 cohorts. ${ }^{16}$ The spatial distribution of tumors and their biofilm status for all three cohorts are depicted in Fig. 1b. Individual study tumor maps are shown in Supplementary Fig. S1b. Each study individually had a significantly higher prevalence of biofilms on right-sided tumors by Fisher's exact test (Fig. 1c; USA $p<0.0001$; MAL1 $p=0.029$; MAL2 $p=0.008$ ), as did the combined analysis of all three studies (Fig. 1d, $p<0.0001$ ). However, the Malaysian cohorts MAL1 and MAL2 had approximately a 3-fold higher prevalence of biofilms on left-sided tumors than the USA cohort (33\% for MAL1, 38\% for MAL2, and $12 \%$ for USA), which may reflect differences in diet/lifestyle, genetics, or the native microbial environment of the two countries. As previously reported for the USA cohort, the biofilm status of paired normal tissues from MAL1 and MAL2 largely matched the biofilm status of the tumors from the same individual and therefore were called concordant pairs; only 2/21 MAL1 and 3/23 MAL2 patients had discordant biofilm scores between their tumor and normal tissues, all 5 of which were cases in which the tumor was biofilm positive but the paired normal tissue was negative. Multivariate logistic regression analysis revealed that tumor stage $[N=74$ cancers, excluding surgical polyps ( 6 from USA; 1 from MAL1)] was not significantly associated with biofilm status after controlling for tumor side (Fig. 1e, right tumors $p>0.48$ for all stages; left tumors $p>0.19$ for all stages).

The concordant tumor-normal biofilm-positive pairs from MAL1 (nine pairs) and MAL2 (ten pairs) were subsequently examined by multi-probe FISH with probes against four taxonomic groups that were previously found to be the dominant bacterial members of biofilms in the USA cohort: phylum Bacteroidetes (including Bacteroides, Parabacteroides, and Prevotella), genus Fusobacterium, family Lachnospiraceae, and the classes Gamma- and Betaproteobacteria. As with the USA cohort, the Malaysian biofilms tended to be polymicrobial, with an abundance of Lachnospiraceae, Bacteroidetes, and Proteobacteria in both tumor and normal tissues from 17/19 patients. Fusobacterium was also detectable in almost all (16/17) tumors with polymicrobial biofilms, ranging from sparse populations (less than 5 bacteria visible in a $200 \mu \mathrm{m} \times$ $200 \mu \mathrm{m}$ field of view) in the majority of tumors to dense blooms in $4 / 17$ of the biofilm-positive tumors (Fig. 1f). In contrast, paired normal tissues were largely devoid of Fusobacterium (Fig. 1f). Sequencing analyses (see subsequent sections) from frozen tissues of the same patients revealed that $F$. nucleatum was often the most abundant fusobacterial species detected in these tissues. The remaining two patients' biofilms consisted exclusively of Proteobacteria in both the tumor and paired normal tissue (representative tumor/normal staining shown in Fig. 1g). Thus, tumors and paired normal tissues were largely concordant with respect to both biofilm status and biofilm composition with the exception of Fusobacterium, which was largely present only in tumor biofilms.

Microbial taxa and functions associated with biofilm status

To complement the results of biofilm quantification, we next sought to identify microbial members associated with biofilmpositive status. Traditional analyses of 16S rRNA gene sequencing data have been limited to genus-level identification. To enhance our taxonomic resolution, 16S rRNA amplicon sequence data sets from USA, MAL1, and MAL2 cohorts were analyzed using Resphera Insight, a high-resolution methodology for species-level characterization (see Methods). ${ }^{24-26}$ We then assessed significant enrichment or depletion of taxa in biofilm-positive vs. biofilmnegative samples.

A limited number of specific species were identified as differentially abundant between biofilm-positive and biofilmnegative tissues (Supplementary Fig. S2). Of the four species, one was additionally found to be enriched in right-sided vs. left- sided samples (Clostridium ramosum), indicating a potential association with geographical location rather than strictly biofilm status (Supplementary Fig. S3). Despite the limited number of differences at the species level between biofilm-positive and biofilm-negative tissues, a meta-analysis of functional in silico predictions by PICRUSt revealed several biofilm-associated functional shifts, including an increase in gene content attributed to cytoskeletal proteins, peptidoglycan biosynthesis, sporulation, peptidases, novobiocin biosynthesis, and ansamycin biosynthesis, as well as a reduction in flagellar assembly genes (Fig. 2a). Examination of taxa contributing most strongly to these functional associations highlighted multiple, differentially abundant families including significant enrichment of Veillonellaceae, Lachnospiraceae, and Coriobacteriaceae that corresponded to the enhanced functions in biofilm-positive samples, whereas a significant decrease in relative abundance of Sphingomonadaceae and a trend towards lower levels of Caulobacteraceae and Enterobacteriaceae contributed to reductions in flagellar assembly (Fig. 2b).

Meta-analysis of microbial associations with CRC status independent of biofilms

We next examined bacterial composition independently of biofilm status in the USA, MAL1, and MAL2 cohorts. For USA and MAL1, CRC samples were compared to both paired normal as well as healthy biopsies, whereas for MAL2 only CRC and paired normal tissues were examined (Fig. 3a). We observed a significant enrichment of the human gut commensal Bacteroides fragilis in CRC compared to both normal flanking tissue and healthy biopsies (Fig. 3b). Additionally, four species known to be oral pathogens were also significantly enriched in CRC tissue: Fusobacterium nucleatum, Parvimonas micra, Peptostreptococcus stomatis, and Gemella morbillorum. Given this strong association between oral pathogens and CRC, we subsequently looked for enrichment of total oral bacteria from the Human Oral Microbiome Database (HOMD), a curated list of bacteria derived from $16 \mathrm{~S}$ rRNA gene sequencing of healthy and diseased oral samples from patients (see Supplementary Table S2 for list of species). ${ }^{27}$ This analysis revealed a robust, significant enrichment of total HOMD bacterial species in CRC tissue compared to both paired normal tissue and healthy biopsies. Enrichment of the species identified above in the tumors was not specific to tumor location; both right-sided tumors and left-sided tumors contributed to the enrichment of $F$. nucleatum, $P$. micra, $P$. stomatis, G. morbillorum, and HOMD species (Supplementary Fig. S4). To further validate these initial findings, we chose samples from the MAL1 cohort and performed qPCR for the 16S rRNA gene for $B$. fragilis and $F$. nucleatum. Read counts from the $16 \mathrm{~S}$ rRNA amplicon sequencing analysis for both $B$. fragilis and $F$. nucleatum significantly correlated with copy numbers from 16S rRNA gene qPCR (Supplementary Fig. S5).

The above analysis was then expanded to include all available $16 \mathrm{~S}$ rRNA gene sequencing data sets on CRC samples, including two additional studies involving CRC versus healthy biopsy samples, 8,15 five studies on CRC versus paired normal tissue, ${ }^{2,3,6,15,28}$ and four stool-based studies. , $^{4,9,28}$ In total, eight data sets were analyzed for differences between CRC and healthy biopsies and/or stool, and eight data sets were analyzed for differences between CRC and paired normal tissue. Table 1 summarizes the country of origin, tissue type (stool vs. tissue biopsy), DNA extraction method, sequencing platform, 16S rRNA gene primers, and original findings of each publication as reported by their respective authors prior to our meta-analysis. Raw data sets were preprocessed using a standardized methodology (see Methods). Collectively, this analysis encompassed populations in North America (U.S., Canada), Europe (France, Germany, Spain) and Asia (China, Vietnam, Malaysia), and represented stools from 481 tumor patients and 271 healthy 

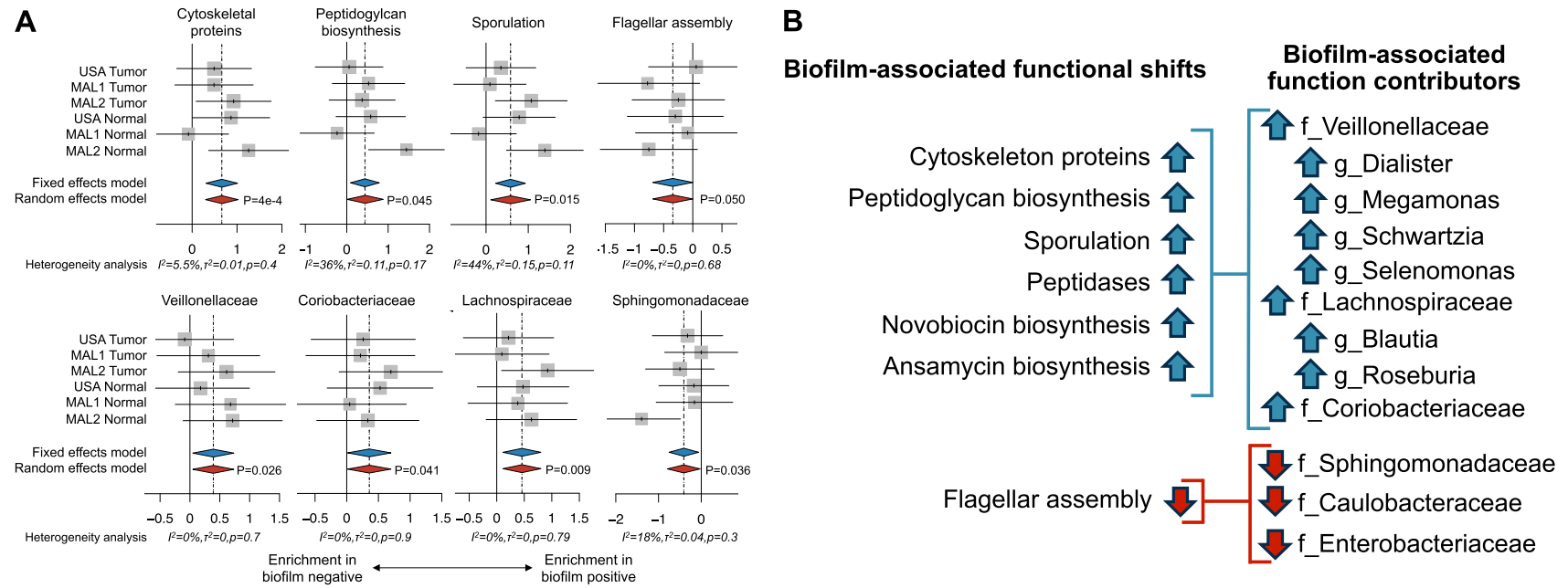

Fig. 2 Microbial associations with biofilm status. Frozen tissue from USA, MAL1, and MAL2 cohorts were characterized by $16 \mathrm{~S}$ rRNA amplicon sequencing. a Top panel: biofilm positive CRC and normal flanking tissues demonstrated several functional shifts in the bacterial composition, including increases in gene content associated with cytoskeletal proteins, peptidoglycan biosynthesis, sporulation, and flagellar assembly based on PICRUSt analysis. Bottom panel: the bacterial families Veillonellaceae, Coriobacteriaceae, and Lachnospiraceae were enriched in biofilm-positive samples, while Sphingomonadaceae was enriched in biofilm-negative samples. Random-effects models with $95 \% \mathrm{Cl}$ above or below 0 (red diamonds) were considered statistically significant. Hedge's $g$ difference statistic is shown on the $X$ axes. The fixed effects model assumes there exists a single effect size shared by all included studies, while the random effects model allows for variation in the effect size from study to study. Heterogeneity analysis includes estimates of $I^{2}$ (percentage of variation reflecting true heterogeneity), $\tau^{2}$ (random-effects between study variance), and $p$-value from Cochran's $Q$ test for heterogeneity. $\mathbf{b}$ The functional alterations due to biofilm status were linked to multiple, differentially abundant families ( $\mathrm{f}$ ) and genera $(\mathrm{g})$ in the biofilms

controls, as well as colon tissues from 379 tumors, 369 paired normal tissues from tumor hosts and 172 biopsies (from 89 patients).

Enrichment of specific species in the initial USA/MAL1/MAL2 cohort analysis was validated in this expanded analysis: $B$. fragilis, F. nucleatum, P. micra, P. stomatis, G. morbillorum, and the total oral microbiome from HOMD (Fig. 4). The statistical significance for each individual microbe from HOMD is provided in Supplementary Table S2. We found these enrichments to be robust to variations in normalization strategy and confidence in specieslevel assignment (Supplementary Fig. S6). The stool studies also supported these findings, although the signal was weaker in comparison to the robust differences seen in tissues (Fig. 4). Although others have reported an enrichment of fusobacterial species and the enterotoxigenic strain of $B$. fragilis in late-stage tumors, ${ }^{29,30}$ we did not observe any consistent changes with respect to $F$. nucleatum, $B$. fragilis, $\mathrm{HOMD}$, or combinations of these species and tumor stage (Supplementary Fig. S7). Conversely, several Bacteroides species (B.vulgatus, B. dorei, and B. stercoris) as well as Faecalibacterium prausnitzii were consistently depleted in CRC compared to healthy biopsies and paired normal tissues (Supplementary Fig. S8).

Not all fusobacterial species are enriched in CRC

The expanded meta-analysis also uncovered significant enrichment of additional fusobacterial species $F$. necrophorum, $F$. periodonticum, and L. trevansanii in CRC samples compared to healthy biopsies (Fig. 5a). However, there were several other species within the fusobacteria phylum that were not enriched in CRC and were in some cases more highly abundant in the healthy tissues. Fusobacteria not associated with CRC included $F$. necrogenes, F. mortiferum, F. varium, and F. ulcerans (Fig. 5b). Additionally, two healthy biopsy patients from Malaysia (MAL1) harbored very high levels of the Fusobacteriaceae member Cetobacterium somerae ( $>20 \%$ relative abundance of all reads; Fig. 5c). Such data highlight the importance of detection down to the species level, as only a subset of the genus Fusobacterium was associated with CRC and an analysis limited to genera would therefore have resulted in a less robust or even non-significant signal. Moreover, the highly abundant intestinal commensal Faecalibacterium prausnitzii was originally classified as Fusobacterium prausnitzii until $2002 ;{ }^{31}$ had this species not been reclassified, it would have strongly confounded genus and higher-level signals associated with CRC.

\section{Compilation of all microbial features of CRC}

Finally, we assessed the combined prevalence of biofilms, $B$. fragilis, and oral microbes from HOMD in order to determine the percentage of CRC tumors with at least one feature of microbial dysbiosis. We selected samples for which data on all three microbial features were available (i.e., samples from the USA, MAL1 and MAL2 cohorts). We defined enrichment of $B$. fragilis as tumors with $B$. fragilis relative abundance $>2 \%$ of overall sequences, and enrichment of HOMD as tumors having HOMD bacteria present at $>10 \%$ of overall sequences; these cut off points excluded $>90 \%$ of healthy biopsies. Tumors harbored the highest percentages of all three features: $49 \%$ were biofilm-positive, $48 \%$ were HOMD positive, and $38 \%$ were B. fragilis positive (Fig. 6a). While a similar percentage of paired normals were biofilm positive ( $44 \%, p=0.721$ by Fisher's exact test compared to tumors), the prevalence of HOMD and $B$. fragilis in paired normal tissues was approximately half of that found in tumors, significantly so for HOMD (HOMD: 24 vs. 48\%, $p=0.009$; B. fragilis: 17 vs. $38 \%, p=$ 0.254 , respectively; Fig. $6 a$ ). Only $2 / 11$ paired normal tissues that were positive for $B$. fragilis had tumors that were below the cut-off value, while similarly $3 / 15$ paired normal tissues that were positive for HOMD had tumors below the HOMD cut-off value. Of the 33 healthy biopsies randomly chosen for sequencing, representing left and right biopsies from 16 patients and one biopsy from the right side of a 17 th patient, only $6 \%$ were biofilm positive (2/33), with both biofilms occurring on right-sided biopsies from the MAL1 cohort (Supplementary Table S1). Three percent of the biopsies were enriched for HOMD organisms, and $0 \%$ were enriched for $B$. fragilis. These percentages were significantly lower 
A
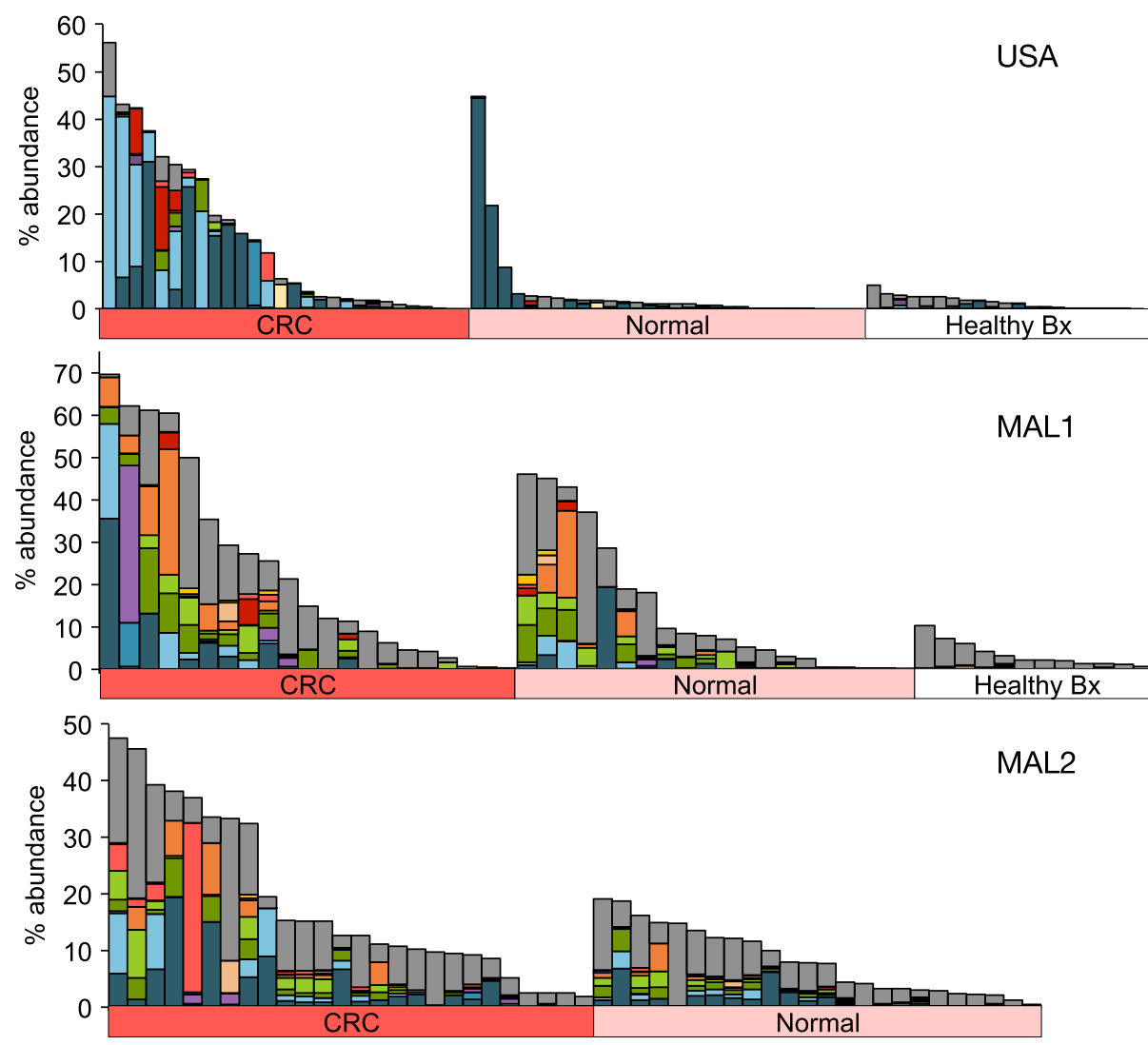

MAL2 $\square$ Other Oral Species

$\square$ Streptococcus mutans

$\square$ Solobacterium moorei

$\square$ Selenomonas sputigena

-Prevotella tannerae

$\square$ Klebsiella pneumoniae

$\square$ Gemella morbillorum

口Peptostreptococcus stomatis

口Parvimonas micra

- Leptotrichia trevisanii

$\square$ Fusobacterium periodonticum

口Fusobacterium nucleatum

$\square$ Fusobacterium necrophorum

$\square$ Bacteroides fragilis

B

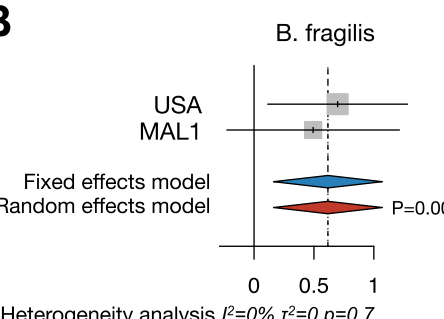

Heterogeneity analysis $l^{2}=0 \%, T^{2}=0, p=0.7$
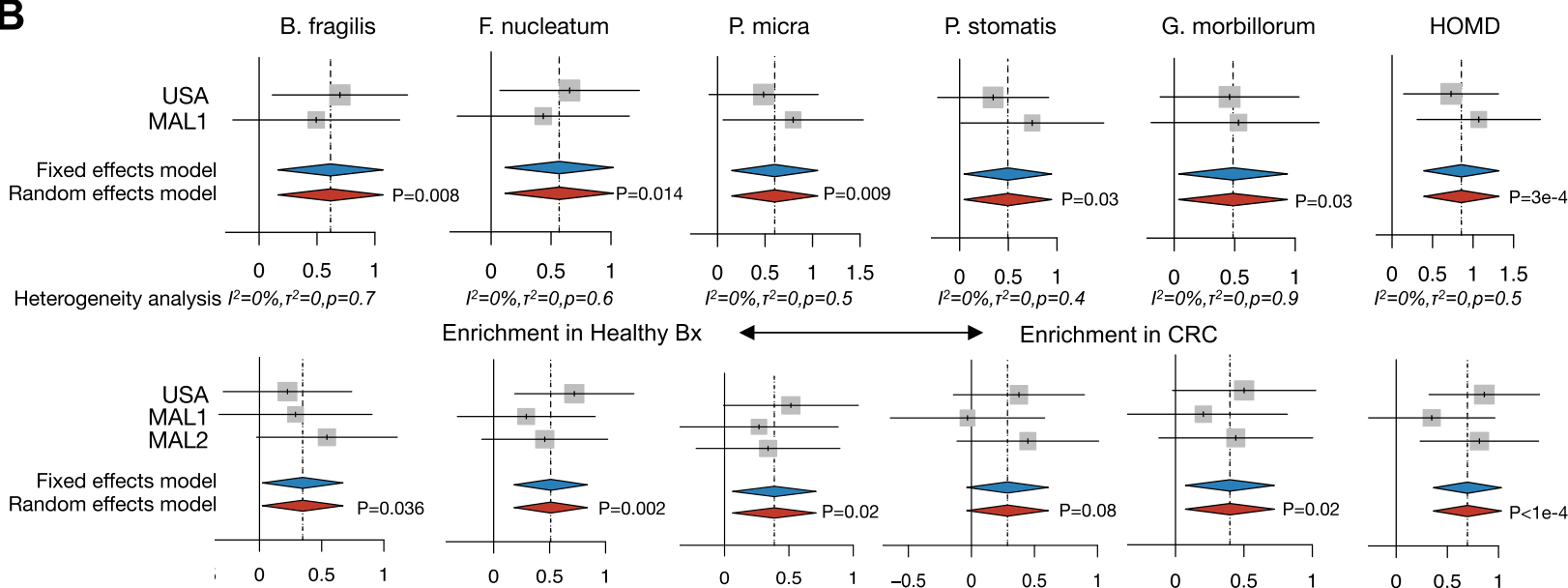

Enrichment in Healthy $B x$
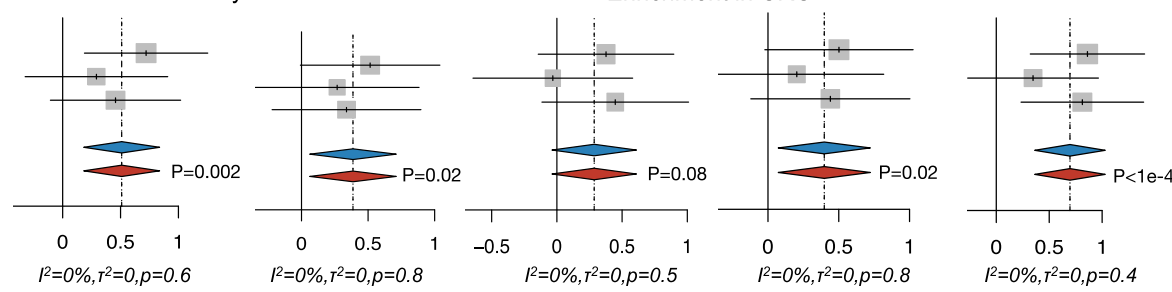

Enrichment in Normal

$\rightarrow$ Enrichment in CRC

Fig. 3 Associations of $B$. fragilis and human oral microbiota with CRC status for USA, MAL1, and MAL2 cohorts. Microbiome profiling with 16S rRNA gene sequencing was applied to tumor (CRC) and paired normal tissues (Normal) from CRC patients for all three cohorts, as well as healthy biopsies (Healthy $\mathrm{Bx}$ ) for USA and MAL1. a Bar charts of microbial sequence relative abundance for $B$. fragilis and bacteria from the Human Oral Microbiome Database (HOMD) from the three 16S rRNA amplicon data sets: USA (top panel), MAL1 (middle panel), and MAL2 (bottom panel). Each vertical bar represents an individual patient. b $B$. fragilis as well as the oral pathogens F. nucleatum, P. micra, P. stomatis, G. morbillorum, and the overall consortia associated with the Human Oral Microbiome Database (HOMD) were all found to be significantly enriched in tumor specimens compared to biopsies from healthy patients without cancer (top panel), and compared to normal flanking tissue (bottom panel). Random-effects models with $95 \% \mathrm{Cl}$ above or below 0 (red diamonds) were considered statistically significant. Hedge's $g$ difference statistic is shown on the $X$ axes. The fixed effects model assumes there exists a single effect size shared by all included studies, while the random effects model allows for variation in the effect size from study to study. Heterogeneity analysis includes estimates of $I^{2}$ (percentage of variation reflecting true heterogeneity), $\tau^{2}$ (random-effects between study variance), and $p$-value from Cochran's $Q$ test for heterogeneity 


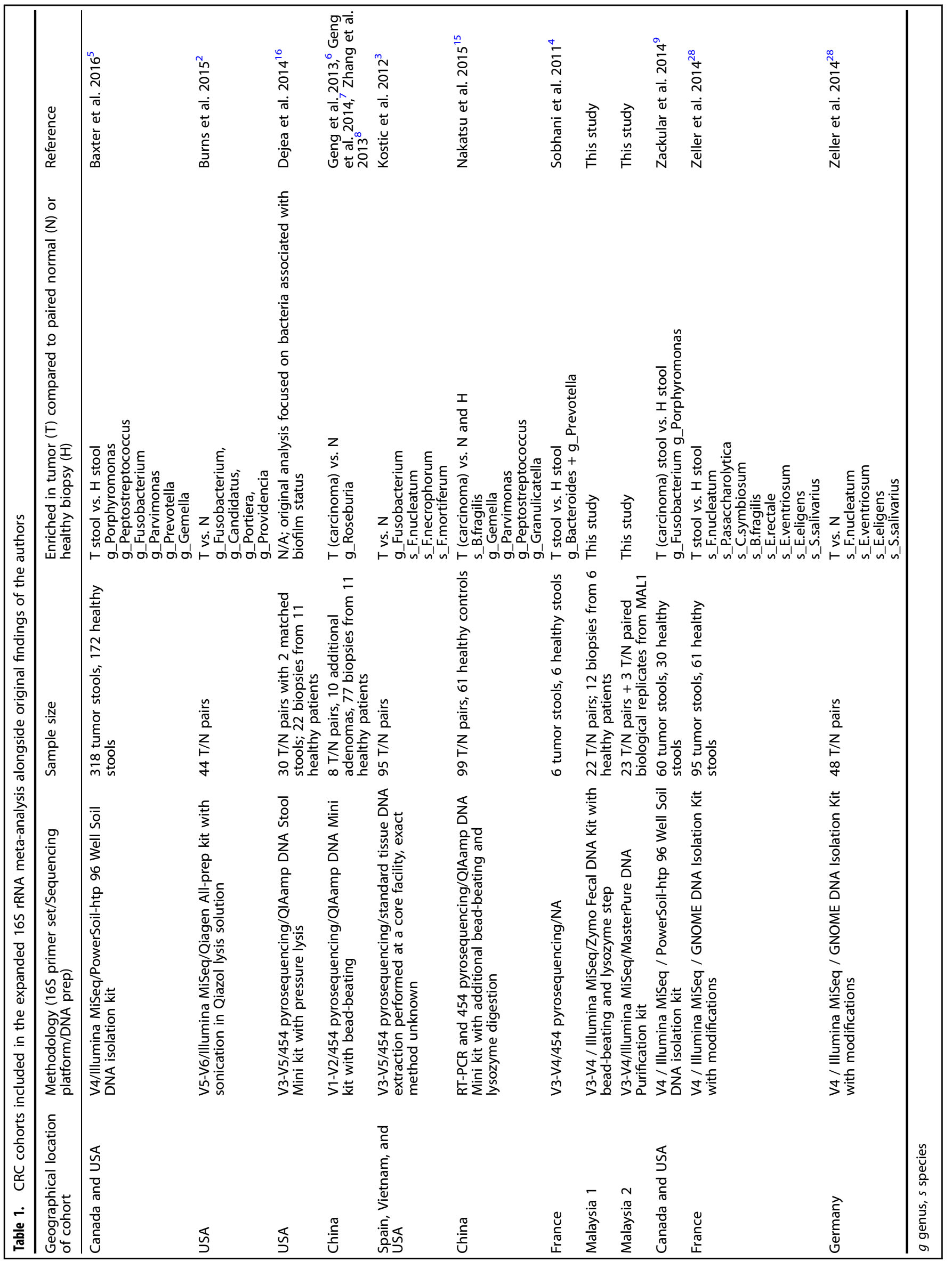


B. fragilis

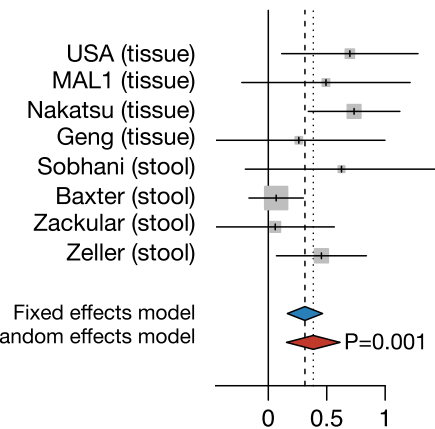

F. nucleatum

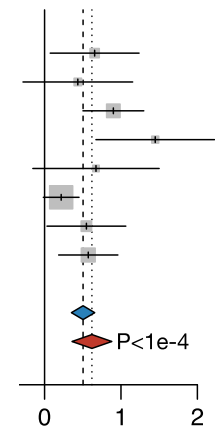

P. micra

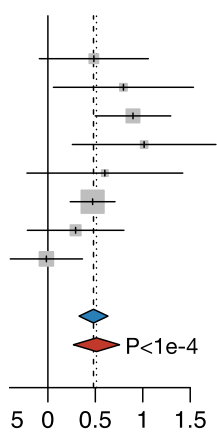

P. stomatis

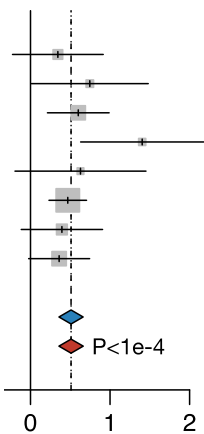

Heterogeneity analysis $I^{2}=44 \%, T^{2}=0.04, p=0.08 \quad l^{2}=56 \%, T^{2}=0.07, p=0.03 \quad l^{2}=50 \%, T^{2}=0.06, p=0.05 \quad l^{2}=0.5 \%, T^{2}=0, p=0.4$

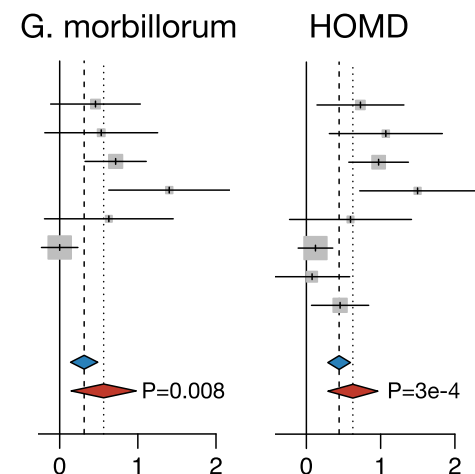

$l^{2}=75 \%, T^{2}=0.18, p<0.01 \quad l^{2}=74 \%, T^{2}=0.15, p<0.01$

Enrichment in Healthy

Enrichment in CRC

B

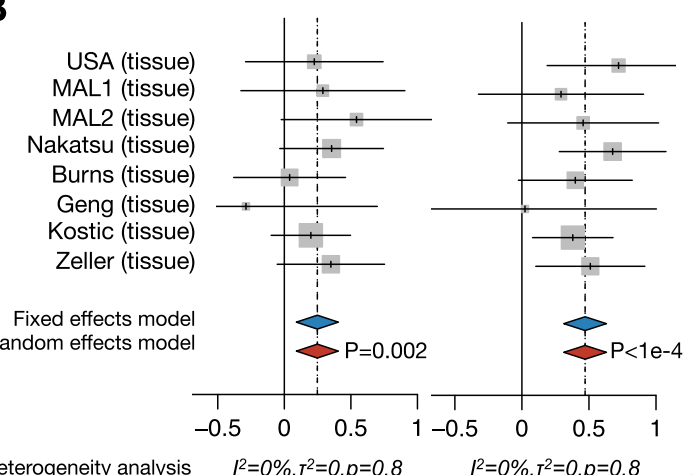

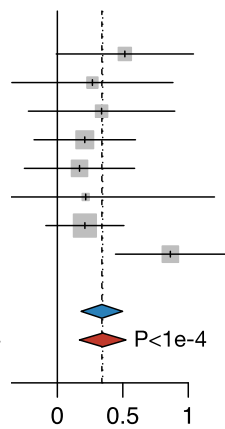

$P^{2}=16 \%, T^{2}=0.01, p=0.3$

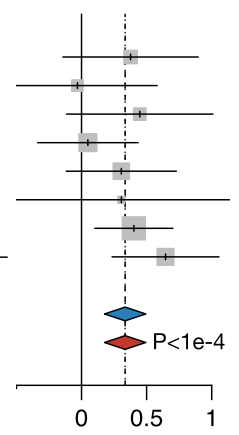

$l^{2}=0 \%, T^{2}=0, p=0.5$

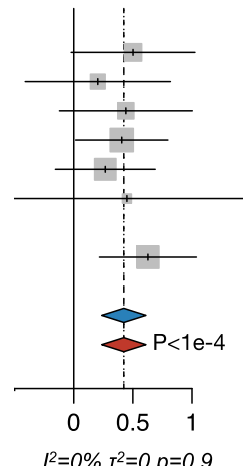

$r^{2}=0 \%, T^{2}=0, p=0.9$

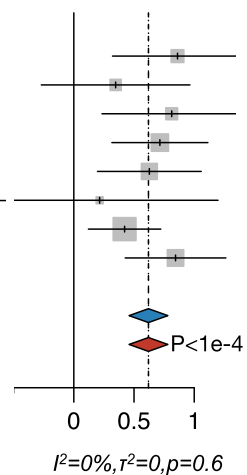

Enrichment in CRC

Fig. 4 Expanded differential relative abundance meta-analysis supports species-level findings. A meta-analysis of a CRC tissue or stool vs. healthy biopsy or stool and $\mathbf{b}$ CRC vs. flanking normal tissues supports the enrichment of $B$. fragilis and oral microbes in CRC. Random-effects models with $95 \% \mathrm{Cl}$ above or below 0 (red diamonds) were considered statistically significant. Hedge's $g$ difference statistic is shown on the $X$ axes. The fixed effects model assumes there exists a single effect size shared by all included studies, while the random effects model allows for variation in the effect size from study to study. Heterogeneity analysis includes estimates of $I^{2}$ (percentage of variation reflecting true heterogeneity), $\tau^{2}$ (random-effects between study variance), and $p$-value from Cochran's $Q$ test for heterogeneity

than the percentages observed for paired normals and tumors for each feature by Fisher's exact test (healthy biopsy vs. paired normal: $p<0.0001$ for biofilms, $p<0.01$ for HOMD, $p<0.01$ for $B$. fragilis; healthy biopsy vs. tumor: $p<0.0001$ for all three features).

In addition to having the highest percentages of each individual microbial feature above, an abundance of tumors $(27 / 63$ or $43 \%$ total) harbored more than one feature ( $41 \%$ single, $35 \%$ two, and $8 \%$ three features) (Fig. 6b). When tumors were analyzed according to their anatomical location, right-sided tumors, not unexpectedly, displayed more biofilm-related categories, while the predominant phenotype of left-sided tumors reflected the cooccurrence of B. fragilis and HOMD (Supplementary Fig. S9). In contrast to the tumors, paired normal tissue contained mostly single features ( $51 \%$ single, $17 \%$ two, and $0 \%$ three features), with the majority of those harboring more than one feature also having tumors with more than one feature $(8 / 11)$. The healthy biopsies contained only single features (6\% biofilm only, 3\% HOMD only) and were otherwise absent of any dysbiosis (91\% no features). Overall, $84 \%$ of tumors harbored at least one measure of microbial dysbiosis associated with CRC status as defined in our metaanalysis, compared to $68 \%$ of paired normal tissues and only $9 \%$ of healthy biopsies.

\section{DISCUSSION}

Overall, our meta-analysis demonstrates that the vast majority $(>80 \%)$ of CRC cases contain aberrant microbial signatures indicative of dysbiosis. Our high-resolution 16S rRNA gene metaanalysis captured species-level taxonomic assignments, allowing for the most detailed map to date of the microbial dysbiosis in CRC. These data support multiple avenues by which bacteria may promote carcinogenesis, including enrichment of a symbiote with enterotoxigenic capabilities ( $B$. fragilis), the emerging role of oral microbes as potentially hostile guests in the gut, and the establishment of polymicrobial, procarcinogenic biofilms. ${ }^{16,32,33}$ Additionally, while specific organisms, such as $B$. fragilis and $F$. nucleatum have been implicated by individual studies using alternative methods such as metagenomic sequencing or qPCR, the present meta-analysis pipeline enabled consistent detection of these species by 16S rRNA gene sequencing-often in cases where the original authors' findings were restricted to genus-level findings-regardless of the sequencing platform, 16S rRNA gene primer set, or DNA extraction method used. Importantly, the microbial features detected in our study were not mutually exclusive and in fact were often found to co-occur in patients in our meta-analysis. However, whether these microbial features are required for the initiation or progression of tumorigenesis or whether they are merely bystanders remains to be elucidated.

$B$. fragilis is a well-studied human gut symbiont, making its enrichment associated with CRC status potentially surprising. However, enterotoxigenic strains of $B$. fragilis (ETBF) harboring the $B$. fragilis toxin (BFT) have long been associated with diarrheal disease and, potentially, inflammatory bowel disease and CRC. ${ }^{30,34}$ Mouse and in vitro studies have shown that BFT likely promotes 
A

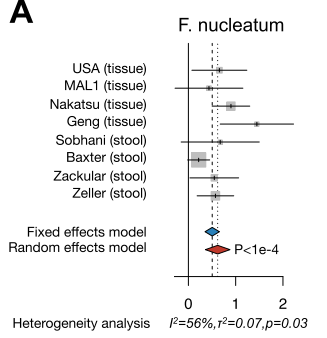

B

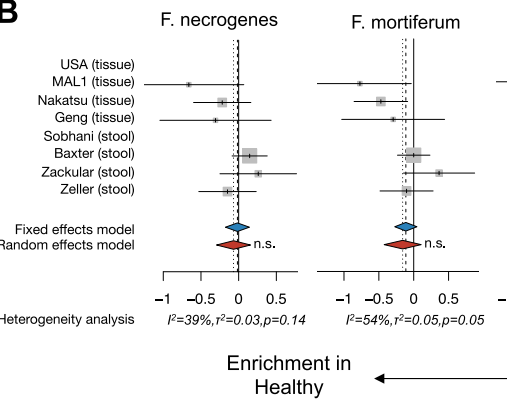

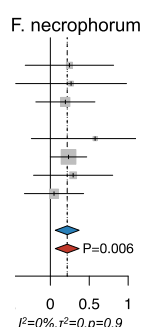
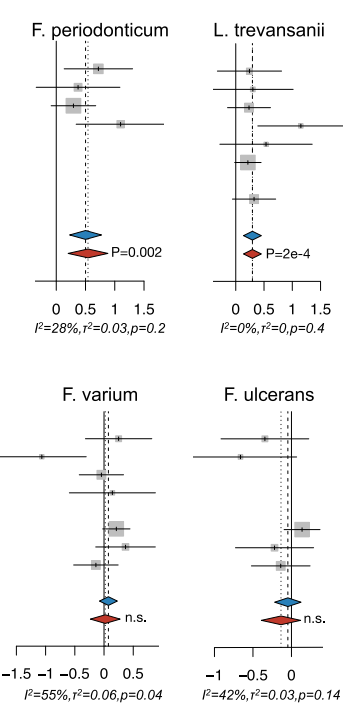

$-1 \quad-0.5 \quad 0$

Enrichment in

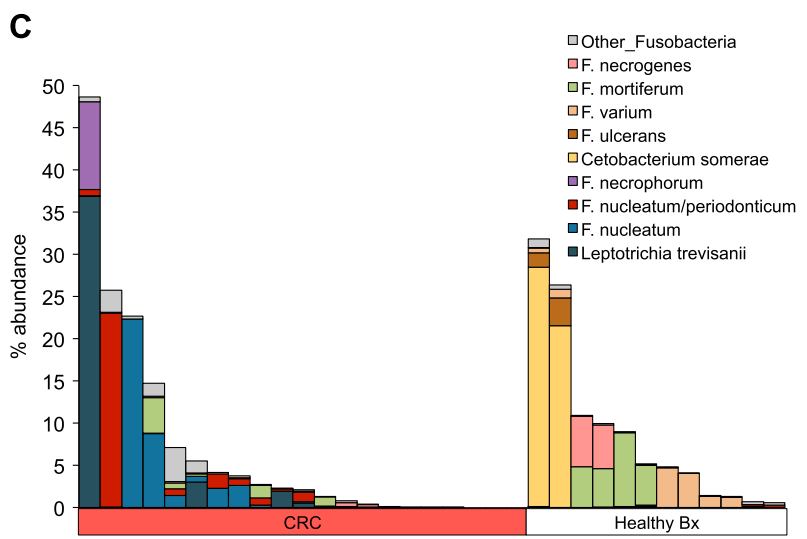

Fig. 5 Not all fusobacterial species are associated with CRC. a In addition to $F$. nucleatum, we detected significant enrichment of $F$. necrophorum, F. periodonticum, and L. trevansanii in CRC tissue or stool compared to healthy biopsies or stool. Hedge's $g$ difference statistic is shown on the $X$ axes. The $F$. periodonticum forest plot includes ambiguous assignments (see Methods). $\mathbf{b}$ Other fusobacterial species were not differentially abundant between CRC and healthy patients. The fixed effects model assumes there exists a single effect size shared by all included studies, while the random effects model allows for variation in the effect size from study to study. Heterogeneity analysis includes estimates of $I^{2}$ (percentage of variation reflecting true heterogeneity), $\tau^{2}$ (random-effects between study variance), and $p$-value from Cochran's $Q$ test for heterogeneity. c Bar chart of all detected species within the fusobacterial phylum and their total sequence relative abundances in the MAL1 cohort. Each vertical bar represents an individual patient. Several healthy Malaysian patients in the MAL1 cohort harbored high levels of fusobacterial species not associated with CRC

tumorigenesis by inducing (1) DNA damage through enhanced spermine oxidase and resulting ROS activity, ${ }^{35}$ and (2) cleavage and subsequent degradation of the tumor suppressor E-cadherin, resulting in increased permeability of the colonic epithelium, enhanced $W n t / \beta$-catenin signaling, and enhanced cellular proliferation. ${ }^{36,37}$ In mouse models, inoculation of ETBF into $\mathrm{Apc}^{\mathrm{Min} /+}$ mice accelerates tumorigenesis in an IL-17-dependent manner. ${ }^{38}$ In patients, bft has been found to be present more frequently in CRC versus controls in both the colon mucosa (>85 vs. $53 \%)^{30}$ and in the stool ( 38 vs. $12 \%$, and 27 vs. $10 \%$ ). ${ }^{39,40}$ Thus, the association between enrichment of $B$. fragilis and CRC observed in our metaanalysis may in fact reflect enrichment of ETBF strains of $B$. fragilis in the gut. Importantly, the enrichment of $B$. fragilis was paralleled by depletion of several other Bacteroides members, which is consistent with reports by others of an overall loss of phylum Bacteroidetes $^{3}$ and more specifically the genus Bacteroides., ${ }^{9,12}$ The selective enrichment of $B$. fragilis may therefore suggest a growth advantage of $B$. fragilis over other Bacteroides species during tumorigenesis, and further highlights the importance of specieslevel resolution.

Our meta-analysis also revealed a robust enrichment of $F$. nucleatum and other oral species in CRC compared to both paired normal and healthy biopsy tissues. These data are consistent with a recent meta-analysis by Shah et al. in stool samples in which Parvimonas micra and two separate unclassified Fusobacterium were reported to be enriched in stools from CRC patients compared to healthy controls. ${ }^{41}$ However, that study was unable to identify $F$. nucleatum specifically, likely due to the signal being weaker in stool compared to tissue and/or the enhanced resolution of our meta-analysis pipeline compared to the strain select tool SS-UP used in that study. $F$. nucleatum has gained increasing notoriety in recent years as a potential pathogen in a number of clinical diseases including gastro-intestinal disorders, cardiovascular disease, adverse pregnancy outcomes, respiratory tract infections, and oropharyngeal infections, where it was first discovered. ${ }^{42}$ Mechanistically, $F$. nucleatum is a ubiquitous oral bacterium that is both highly adherent and invasive, a property attributed to virulence proteins including the adhesion and invasion protein, FadA, and the galactose-inhibitable adhesion protein Fap2. ${ }^{43-45}$ More recently, secreted or surface FadA was found to bind to E-cadherin and induce $W n t / \beta$-catenin signaling in the colons of mice, where $F$. nucleatum accelerated tumorigenesis. ${ }^{46,47}$ Immune mechanisms including myeloid cell infiltration, the balance between FOXP3 ${ }^{\text {hi }}$ and FOXP3 ${ }^{\text {lo }}$ Treg populations, and checkpoint molecule (TIGIT) blockade may modulate F. nucleatumassociated carcinogenesis. ${ }^{4-50}$

However, Fusobacterium are enriched in only a subset of tumors and are present at a much lower abundance in paired normal

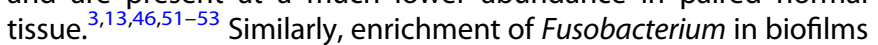
occurs in only a subset of tumors and is often undetectable in paired normal tissues, ${ }^{16}$ findings that our additional cohort of Malaysian patients and meta-analyses upheld. As most carcinogenic mechanisms are predicted to be elevated in adjacent normal tissue as well as the tumor itself, in line with the cancer hypothesis that it takes years or even decades for cancer to develop within normal colon tissue, these data question whether $F$. nucleatum is an important initiator of carcinogenesis. Instead, $F$. nucleatum may be uniquely suited to grow and contribute to tumor progression in an established tumor microenvironment, due to the microbe's highly adherent and invasive nature that could exploit a compromised colonic epithelial cell (CEC) layer. Further, as Kostic et al. have proposed, the asaccharolytic metabolism of $F$. nucleatum would not compete for glucose consumption by the tumor, and the anaerobic nature of $F$. nucleatum would enable it to tolerate the hypoxic tumor environment. ${ }^{47} F$. nucleatum may even provide a growth advantage for the tumor, due to the bacteria's ability to inhibit NK cell-mediated tumor cell death via binding of the bacterial protein Fap2 to one of the NK cell inhibitory receptors, TIGIT. $^{50}$ The procarcinogenic capability of $F$. nucleatum in the gut may be 
A

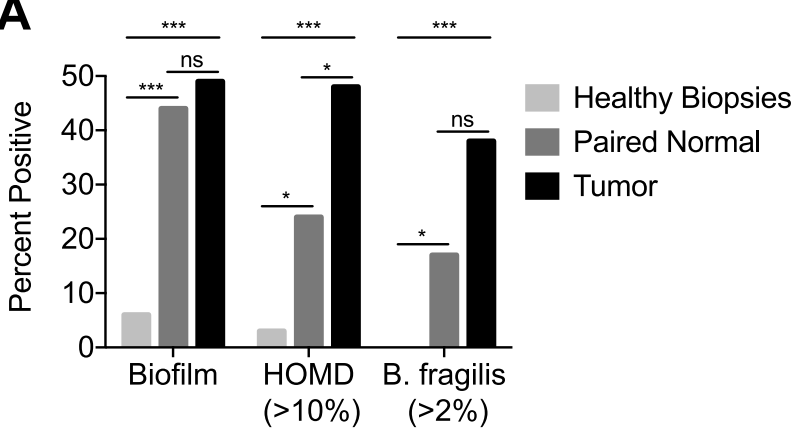

Biofilm only

B. fragilis only

HOMD only

Biofilm + B. fragilis

Biofilm + HOMD

B. fragilis + HOMD

Biofilm + B. fragilis + HOMD

None

B

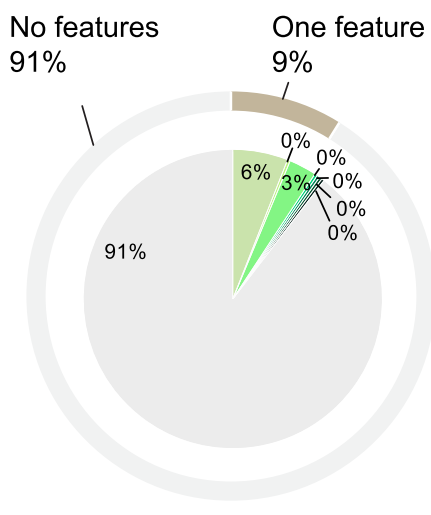

Two

Healthy Biopsies $=33$

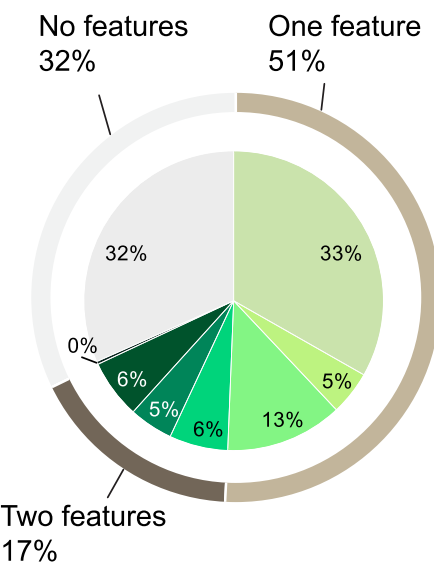

Paired Normal $=63$

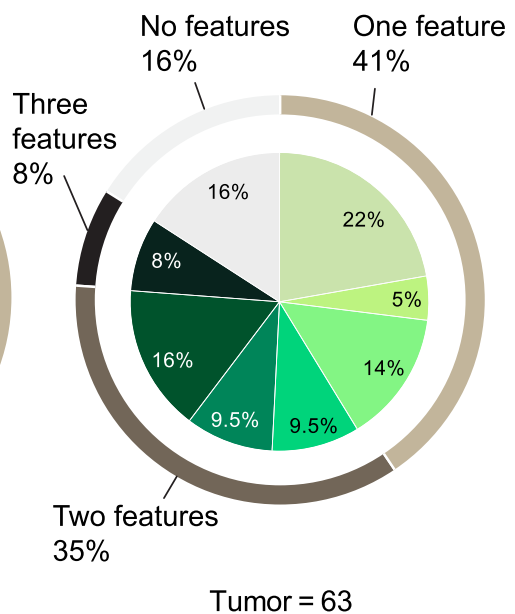

Fig. 6 Combined prevalence of CRC-associated microbial features in USA, MAL1, and MAL2 cohorts. Positive status for B. fragilis and the human oral microbes from the HOMD database were defined as a relative abundance $>2$ and $>10 \%$ of all $16 \mathrm{~S}$ rRNA gene sequences, respectively. a Percentage of healthy biopsies, paired normal, and tumor tissues that were positive for each microbial feature (biofilms, HOMD, and $B$. fragilis). Statistics shown are Fisher's exact test. ${ }^{*} p<0.01,{ }^{* * *} p<0.001$. b Percentage and overlap of healthy biopsies, paired normal, and tumor tissues harboring one, two, three or no microbial features

highly strain-dependent, as some strains have been resistant to colonization in mouse gut and required daily inoculation ${ }^{47}$ while others have been found to colonize readily. ${ }^{49}$

F. nucleatum is frequently found to co-occur with other oral pathogens in $\mathrm{CRC}^{53}$ and can coaggregate with members of all genera in vitro. ${ }^{54}$ As such, $F$. nucleatum may be a bridging organism that assists in the colonization of other microbes through its myriad adhesins, which allow other microbes to adhere to and interact with $F$. nucleatum. ${ }^{42,55}$ Our meta-analysis revealed consistent elevations in not only $F$. nucleatum but also several other oral pathogens (e.g., P. micra and P. stomatis) in CRC compared to paired normal tissue and healthy biopsy controls. A variety of other oral microbes were frequently detected as well, including several Fusobacteriaceae that were enriched in normal flanking tissues compared to CRC from Malaysian patients. Flynn et al. have elegantly proposed a polymicrobial model in which several oral pathogens, reliant on each other for survival, may establish a niche in the gut that may contribute to tumorigenesis due to similarities between the gut and the oral environment such as similar $\mathrm{pH}$ and propensity to form biofilms. ${ }^{33}$ Initiation or progression of tumorigenesis becomes beneficial to these bacteria because of increased nutrients from local inflammatory responses. These organisms include Fusobacterium, Parvimonas, and Peptostreptococcus species found to be enriched in CRC in our metaanalysis, as well as Porphyromonas, Prevotella, and Gemella species enriched in other studies. $5,9,15$

While it is tempting to speculate that biofilms from the oral cavity containing $F$. nucleatum seed the gut biofilms that we have observed in a substantial number of CRC cases, this likely would only account for a subset of biofilm formation events. Sparsely populated ( $<5$ bacteria per $200 \mu \mathrm{m}^{2}$ area) Fusobacterium could be detected in almost all of the biofilms from Malaysia by FISH, but dense fusobacterial aggregates were abundant in only $25 \%$ of the tumor biofilms, were infrequently detected in biofilms on paired normal tissue, and were not detected on any healthy biopsies. By sequencing, approximately only one-third of biofilm-positive tumors were enriched for oral consortia derived from the HOMD, while $<10 \%$ of biofilm-positive paired normal tissues and $0 \%$ of biofilm-positive healthy biopsies $(N=2)$ contained HOMD levels of an appreciable relative abundance. These data suggest that oral bacteria such as $F$. nucleatum are not required for biofilm formation in the gut and that therefore the initiating species may be different from that of oral biofilms.

Certainly, further studies on how and why biofilms form in the human gastrointestinal tract are necessary. The data thus far support a role for biofilms in causality of a subset of CRC, particularly right-sided CRC, where nearly $100 \%$ of tumors and paired normal tissue have been found to be covered in invasive, polymicrobial biofilms adjacent to the CEC layer. In comparison, a range of $13-35 \%$ of healthy screening colonoscopy patients have been reported to harbor colonic biofilms, with equivalent percentages of biofilms on the right and left sides. ${ }^{16,23}$ Notably, the prevalence of biofilms in the healthy biopsies in the present meta-analysis was lower than expected (6\%); however, separating these samples by cohort revealed that $2 / 12$ MAL1 biopsies (17\%) were biofilm positive, while the 21 USA biopsies chosen for sequencing were part of a larger cohort previously published in which $15 / 120$ biopsies (13\%) were biofilm positive. ${ }^{16}$ Clearly there 
is a need for a much larger study of biofilms in healthy screening colonoscopy individuals to resolve the true estimated prevalence, but the current estimates still point to a much higher rate of biofilms on proximal tumors than on proximal biopsies. The potential procarcinogenic mechanisms of biofilms elucidated thus far include production of the polyamine (N)1, (N)12-diacetylspermine $^{56}$ and induction of a pro-inflammatory response involving enhanced epithelial levels of IL-6, phospho-STAT3, and increased crypt epithelial cell proliferation. ${ }^{16}$ Importantly, one cannot predict biofilm status from sequencing data alone. For biofilm-negative tissues, sequencing likely detected bacteria enmeshed in the loose, permissive outer mucus layer, a distance from the CECs, whereas, for biofilm-positive tissues, bacteria invading the dense, restrictive mucus layer adjacent to the CECs were also detected. Thus, the limited changes in bacterial composition that we observed between biofilm-positive and biofilm-negative samples suggest that the close proximity of the bacteria to the CEC layer and changes in their function within a biofilm (Fig. 2) may be more important to changes in CRC biology than the composition of the biofilm itself.

Overall, our meta-analysis of microbial features in CRC confirms three separate but partially overlapping dysbiotic mechanisms: enrichment of $B$. fragilis, enrichment of oral microbes such as $F$. nucleatum, and a high prevalence of invasive, polymicrobial biofilms. Strengths of the $16 \mathrm{~S}$ rRNA gene meta-analysis in particular include the fact that these commonalities were found in patients from several different countries in North America, Europe, and Asia, using a wide range of sample preparation techniques and 16S rRNA gene sequencing technologies. The implementation of a high-resolution, consistent analysis methodology greatly improved our ability to detect species-level changes compared to the original authors' findings, which were largely restricted to genus-level associations (see Fig. 4 vs. Table 1), as well as compared to the recent stool meta-analysis by Shah et al. ${ }^{41}$ Additionally, samples from not only tumors and paired normal tissue but also healthy biopsies and stool samples were included in the analysis. A similar strength of signal was obtained when comparing CRC vs. paired normal (tissues only) or CRC vs. healthy (tissues and stool), although the latter displayed more heterogeneity by $l^{2}$ values. However, the stool samples displayed weaker associations than tissues, suggesting that a tissue-based approach would be required for potential biomarker studies involving the identified microbial features. Weaknesses include the fact that the present analysis did not take into account the concepts of mucosal-associated bacterial co-abundance groups ${ }^{57}$ or metacommunities, ${ }^{15}$ which may represent another layer of complexity in the microbiome-CRC hypothesis, although our analysis of total HOMD microbes likely parallels a metacommunity phylotype in the latter study. Furthermore, we did not analyze expression of virulence factors, which are an important nuance of bacterial genetics. Despite these limitations, our data strongly support a clear association between multiple, non-mutually exclusive forms of microbial dysbiosis and a substantial portion of CRC cases (>80\%). While we should be cautious in attributing the evolution of carcinogenesis to microbes, defining the subset of patients that may be at risk of microbiome-associated CRC tumorigenesis could be a major turning point in CRC prevention, detection, and treatment. Prospective studies will be necessary to confirm that biofilms, B. fragilis, and oral pathogens confer an increased risk of CRC.

\section{METHODS}

\section{Ethical statement}

This study was approved by the University of Malaya Medical Centre (UMMC, Kuala Lumpur, Malaysia) Medical Ethics Committee (Ref No. 1066.38) and the Johns Hopkins Institutional Review Board. All samples were obtained in accordance with the Health Insurance Portability and
Accountability Act. Blank consent forms for the US and Malaysian cohorts (the latter provided in Malay and English) are available in the Supplementary Information. This research was conducted in accordance to all relevant guidelines and procedures.

\section{Sample collection}

The approach to collection of colon tissues for the USA, MAL1 and MAL2 cohorts has been previously described. ${ }^{16}$ The proximal colon through the hepatic flexure was defined as right colon, and distal to the hepatic flexure as left colon. Samples from MAL1 and MAL2 were maintained as independent cohorts because 16S rRNA amplicon sequencing was performed at different facilities with different methodologies for the two sets of samples. Individuals who had received pre-operative radiation, chemotherapy or had a personal history of CRC or inflammatory bowel disease were excluded. All patients underwent a standard mechanical bowel preparation. Standard pre-operative intravenous, but not oral, antibiotics were administered in all surgical cases. Demographic and histopathology information of the study subjects are summarized in Supplementary Table S1.

Study data were collected and managed using REDCap electronic data capture tools ${ }^{58}$ hosted at Johns Hopkins University.

\section{FISH analysis of biofilms}

FISH was performed on Carnoy's-fixed, paraffin-embedded tissue sections from the Malaysian patients as previously described, with minor modifications ${ }^{16}$ (see SI Methods). Samples were screened in a randomized, blinded fashion.

\section{S rRNA gene Illumina library generation and sequencing}

For the MAL1 cohort, a total of 54 samples were evaluated for sequencing, including paired left-right biopsies from six healthy patients, paired tumor/ normal samples from 20 CRC patients, and an additional two unpaired tumors from two CRC patients. One polyp was sequenced but excluded from analysis. DNA was extracted using the ZR Fecal DNA MiniPrep kit (Zymo Research) with modifications (see SI Methods). High-throughput next-generation sequencing of the V3-V4 hypervariable region of the $16 \mathrm{~S}$ rRNA gene was performed using 319 F (5'-ACTCCTACGGGAGGCAGCAG-3') and 806 R ( $5^{\prime}$-GGACTACHVGGGTWTCTAAT-3') universal primers containing a linker sequence required for Illumina MiSeq $300 \mathrm{bp}$ paired-end sequencing and a 12-bp heterogeneity-spacer index sequence. ${ }^{59,60}$

For the MAL2 cohort, a total of 46 samples, including paired tumor/ normal colon tissue samples from 19 CRC patients, two unpaired tumors from CRC patients, and biological replicates of three MAL1 paired tumor/ normal samples were evaluated. Removal of the biological replicates from either MAL1 or MAL2 did not influence statistical significance of the analysis (Supplementary Fig. S10). DNA was extracted using the MasterPure DNA Purification Kit (Epicentre/lllumina). The V3-V4 region of the 16S rRNA gene was amplified using S-D-Bact-0341-b-S-17 forward (5'NNNNCCTACGGGNGGCWGCAG-3') and S-D-Bact-0785-a-A-21 reverse (5'GACTACHVGGGTATCTAATCC- $3^{\prime}$ ) primers $^{61}$ designed to include the Illumina-compatible adapters. ${ }^{62}$ SI Methods contain additional details of library generation and sequencing.

\section{Analysis of all 16S rRNA amplicon sequence data sets}

Data sets generated utilizing paired-end sequencing on the Illumina platform were first processed as follows: raw paired-end reads were merged into consensus fragments by $\mathrm{FLASH}^{63}$ requiring a minimum $20 \mathrm{bp}$ overlap with $5 \%$ maximum mismatch density, and subsequently filtered for quality (targeting error rates $<1 \%$ ) and length (minimum $150 \mathrm{bp}$ ) using Trimmomatic $^{64}$ and QIIME. ${ }^{65,66}$ Spurious hits to the PhiX control genome were identified using BLASTN and removed.

Data sets generated utilizing Roche/454 sequencing were first preprocessed as follows: sequences were de-multiplexed using $5^{\prime}$ barcode identifiers and filtered for quality and length in QIIME ${ }^{65,66}$ requiring: (i) trimming of the first $15 \mathrm{bp}$ window with a mean Phred quality score below 24, (ii) a maximum homopolymer run of eight nucleotides, and (iii) a minimum final length of $150 \mathrm{bp}$. Passing sequences were error-corrected using Acacia with default parameters. ${ }^{67}$

Resulting sequences from all sequencing technologies were then trimmed of their associated primers, evaluated for chimeras with UCLUST (de novo mode), ${ }^{68}$ and screened for human-associated contaminant using Bowtie ${ }^{69}$ searches of NCBI Homo sapiens Annotation Release 106 followed 
by a BLASTN search against the GreenGenes $16 \mathrm{~S}$ database $(\mathrm{v} 13.05)^{70}$ to identify unaligned host-associated sequences. Reads assigned to chloroplast or mitochondrial contaminants by the RDP classifier $^{71}$ with a minimum confidence of $50 \%$ were removed.

High-quality $16 \mathrm{~S}$ sequences were assigned to a high-resolution taxonomic lineage using Resphera Insight (Baltimore, MD). ${ }^{24-26}$ Sequences were also analyzed by PICRUSt ${ }^{72}$ to infer functional content. Species associated with the human oral tract were determined from the HOMD. ${ }^{27}$

Details of Resphera Insight speciation validation, benchmarking studies and statistical analyses are available in SI Methods.

\section{Code availability}

Open source R code used for meta-analyses is available from the authors upon request.

\section{Data availability}

Raw sequences from new cohorts (MAL1 and MAL2) have been deposited in the NCBI SRA repository (BioProject accession no. PRJNA325649 and PRJNA325650). Primary sequencing data are available upon request.

\section{ACKNOWLEDGEMENTS}

This work was supported by the National Institutes of Health through Grants R01 CA151393 (to CLS), P30 DK089502 (Gl Core), P30 CA006973 (Sidney Kimmel Comprehensive Cancer Center core), P50 CA062924 (SPORE in Gastrointestinal Cancers), JHU Institute for Cancer Immunotherapy, JHU SOM Department of Medicine, JHU SOM Department of Surgery, the Cancer Research Institute/Fight Colorectal Cancer, Swami Institute for International Medical Education (SIIME) Award (to CLS and ECW), University of Malaya Research Grant RP016A-13HTM (to JV), and an $\mathrm{NIH}$ Shared Instrumentation Grant S100D016374 for the Zeiss 780 LSM confocal in the JHU Microscope Facility.

\section{AUTHOR CONTRIBUTIONS}

C.L.S. designed research. J.L.D., C.M.D., P.F., E.F.M., K.T., H.M.G. performed research. J.V. contributed new reagents or analytic tools. J.L.D., J.R.W., E.F.M., M.F.L., K.T., J.W.W., and C.L.S. analyzed data. T.I., A.C.R., E.C.W., K.L.G., H.Y.G., S.K., J.W.W. assisted in the set up, recruitment, or handling of clinical specimens. J.L.D., J.R.W., C.L.S. wrote the paper with input from all authors.

\section{ADDITIONAL INFORMATION}

Supplementary information accompanies the paper on the npj Biofilms and Microbiomes website (https://doi.org/10.1038/s41522-017-0040-3).

Competing interests: J.R.W. is the founder of Resphera Biosciences and has an equity position in the company. The other authors declare that they have no competing financial interests.

Publisher's note: Springer Nature remains neutral with regard to jurisdictional claims in published maps and institutional affiliations.

\section{REFERENCES}

1. Boyle, P. \& Langman, J. S. ABC of colorectal cancer: epidemiology. BMJ 321, 805-808 (2000)

2. Burns, M. B., Lynch, J., Starr, T. K., Knights, D. \& Blekhman, R. Virulence genes are a signature of the microbiome in the colorectal tumor microenvironment. Genome Med. 7, 55 (2015).

3. Kostic, A. D. et al. Genomic analysis identifies association of Fusobacterium with colorectal carcinoma. Genome Res. 22, 292-298 (2012).

4. Sobhani, I. et al. Microbial dysbiosis in colorectal cancer (CRC) patients. PLoS One 6, e16393 (2011)

5. Baxter, N. T., Ruffin, M. T. T., Rogers, M. A. \& Schloss, P. D. Microbiota-based model improves the sensitivity of fecal immunochemical test for detecting colonic lesions. Genome Med. 8, 37 (2016).

6. Geng, J., Fan, H., Tang, X., Zhai, H. \& Zhang, Z. Diversified pattern of the human colorectal cancer microbiome. Gut Pathog. 5, 2 (2013).

7. Geng, J. et al. Co-occurrence of driver and passenger bacteria in human colorectal cancer. Gut Pathog. 6, 26 (2014).

8. Zhang, Z. et al. Spatial heterogeneity and co-occurrence patterns of human mucosal-associated intestinal microbiota. ISME J. 8, 881-893 (2014).
9. Zackular, J. P., Rogers, M. A., Ruffin, M. T. T. \& Schloss, P. D. The human gut microbiome as a screening tool for colorectal cancer. Cancer Prev. Res. (Phila.) 7 , 1112-1121 (2014).

10. Gao, Z., Guo, B., Gao, R., Zhu, Q. \& Qin, H. Microbiota disbiosis is associated with colorectal cancer. Front. Microbiol. 6, 20 (2015).

11. Wang, T. et al. Structural segregation of gut microbiota between colorectal cancer patients and healthy volunteers. ISME J 6, 320-329 (2012).

12. Weir, T. L. et al. Stool microbiome and metabolome differences between colorectal cancer patients and healthy adults. PLoS One 8, e70803 (2013).

13. Marchesi, J. R. et al. Towards the human colorectal cancer microbiome. PLoS One 6. e20447 (2011)

14. Mira-Pascual, L. et al. Microbial mucosal colonic shifts associated with the development of colorectal cancer reveal the presence of different bacterial and archaeal biomarkers. J. Gastroenterol. 50, 167-179 (2015).

15. Nakatsu, G. et al. Gut mucosal microbiome across stages of colorectal carcinogenesis. Nat. Commun. 6, 8727 (2015).

16. Dejea, C. M. et al. Microbiota organization is a distinct feature of proximal colorectal cancers. Proc. Natl. Acad. Sci. USA 111, 18321-18326 (2014).

17. Eckburg, P. B. et al. Diversity of the human intestinal microbial flora. Science $\mathbf{3 0 8}$, 1635-1638 (2005).

18. Costerton, J. W., Stewart, P. S. \& Greenberg, E. P. Bacterial biofilms: a common cause of persistent infections. Science 284, 1318-1322 (1999).

19. Bjarnsholt, T. et al. The in vivo biofilm. Trends Microbiol. 21, 466-474 (2013).

20. Worlitzsch, D. et al. Effects of reduced mucus oxygen concentration in airway Pseudomonas infections of cystic fibrosis patients. J. Clin. Invest. 109, 317-325 (2002).

21. Derrien, $M$. et al. Mucin-bacterial interactions in the human oral cavity and digestive tract. Gut Microbes 1, 254-268 (2010).

22. Palestrant, D. et al. Microbial biofilms in the gut: visualization by electron microscopy and by acridine orange staining. Ultrastruct. Pathol. 28, 23-27 (2004).

23. Swidsinski, A., Weber, J., Loening-Baucke, V., Hale, L. P. \& Lochs, H. Spatial organization and composition of the mucosal flora in patients with inflammatory bowel disease. J. Clin. Microbiol. 43, 3380-3389 (2005).

24. Daquigan, N., Grim, C. J., White, J. R., Hanes, D. E. \& Jarvis, K. G. Early recovery of salmonella from food using a 6-hour non-selective pre-enrichment and reformulation of tetrathionate broth. Front. Microbiol. 7, 2103 (2016).

25. Ottesen, A. et al. Enrichment dynamics of Listeria monocytogenes and the associated microbiome from naturally contaminated ice cream linked to a listeriosis outbreak. BMC Microbiol. 16, 275 (2016).

26. Abernethy, M. G. et al. Urinary microbiome and cytokine levels in women with interstitial cystitis. Obstet. Gynecol. 129, 500-506 (2017).

27. Chen, T. et al. The human oral microbiome database: a web accessible resource for investigating oral microbe taxonomic and genomic information. Database (Oxford) 2010, baq013 (2010).

28. Zeller, G. et al. Potential of fecal microbiota for early-stage detection of colorectal cancer. Mol. Syst. Biol. 10, 766 (2014).

29. Viljoen, K. S., Dakshinamurthy, A., Goldberg, P. \& Blackburn, J. M. Quantitative profiling of colorectal cancer-associated bacteria reveals associations between fusobacterium spp., enterotoxigenic Bacteroides fragilis (ETBF) and clinicopathological features of colorectal cancer. PLoS One 10, e0119462 (2015).

30. Boleij, A. et al. The Bacteroides fragilis toxin gene is prevalent in the colon mucosa of colorectal cancer patients. Clin. Infect. Dis. 60, 208-215 (2015).

31. Duncan, S. H., Hold, G. L., Harmsen, H. J., Stewart, C. S. \& Flint, H. J. Growth requirements and fermentation products of Fusobacterium prausnitzii, and a proposal to reclassify it as Faecalibacterium prausnitzii gen. nov., comb. nov. Int J. Syst. Evol. Microbiol. 52, 2141-2146 (2002)

32. Sears, C. L., Geis, A. L. \& Housseau, F. Bacteroides fragilis subverts mucosal biology: from symbiont to colon carcinogenesis. J. Clin. Invest. 124, 4166-4172 (2014).

33. Flynn, K. J., Baxter, N. T. \& Schloss, P. D. Metabolic and community synergy of oral bacteria in colorectal cancer. mSphere 1, e00102-16 (2016).

34. Sears, C. L. Enterotoxigenic Bacteroides fragilis: a rogue among symbiotes. Clin. Microbiol. Rev. 22, 349-369 (2009).

35. Goodwin, A. C. et al. Polyamine catabolism contributes to enterotoxigenic Bac teroides fragilis-induced colon tumorigenesis. Proc. Natl. Acad. Sci. USA 108 15354-15359 (2011).

36. Wu, S., Lim, K. C., Huang, J., Saidi, R. F. \& Sears, C. L. Bacteroides fragilis enterotoxin cleaves the zonula adherens protein, E-cadherin. Proc. Natl. Acad. Sci. USA 95, 14979-14984 (1998).

37. Wu, S., Morin, P. J., Maouyo, D. \& Sears, C. L. Bacteroides fragilis enterotoxin induces c-Myc expression and cellular proliferation. Gastroenterology 124 392-400 (2003).

38. $\mathrm{Wu}, \mathrm{S}$. et al. A human colonic commensal promotes colon tumorigenesis via activation of T helper type $17 \mathrm{~T}$ cell responses. Nat. Med. 15, 1016-1022 (2009). 
39. Toprak, N. U. et al. A possible role of Bacteroides fragilis enterotoxin in the aetiology of colorectal cancer. Clin. Microbiol. Infect. 12, 782-786 (2006).

40. Keenan, J. I. et al. Screening for enterotoxigenic Bacteroides fragilis in stool samples. Anaerobe 40, 50-53 (2016).

41. Shah, M. S. et al. Leveraging sequence-based faecal microbial community survey data to identify a composite biomarker for colorectal cancer. Gut https://doi.org/ 10.1136/gutjnl-2016-313189 (2017).

42. Han, Y. W. Fusobacterium nucleatum: a commensal-turned pathogen. Curr. Opin. Microbiol. 23, 141-147 (2015).

43. Han, Y. W. et al. Identification and characterization of a novel adhesin unique to oral fusobacteria. J. Bacteriol. 187, 5330-5340 (2005).

44. Han, Y. W. et al. Interactions between periodontal bacteria and human oral epithelial cells: Fusobacterium nucleatum adheres to and invades epithelial cells. Infect. Immun. 68, 3140-3146 (2000).

45. Coppenhagen-Glazer, S. et al. Fap2 of Fusobacterium nucleatum is a galactoseinhibitable adhesin involved in coaggregation, cell adhesion, and preterm birth. Infect. Immun. 83, 1104-1113 (2015).

46. Rubinstein, M. R. et al. Fusobacterium nucleatum promotes colorectal carcinogenesis by modulating E-cadherin/beta-catenin signaling via its FadA adhesin. Cell Host Microbe 14, 195-206 (2013).

47. Kostic, A. D. et al. Fusobacterium nucleatum potentiates intestinal tumorigenesis and modulates the tumor-immune microenvironment. Cell Host Microbe 14, 207-215 (2013).

48. Saito, T. et al. Two FOXP3(+)CD4(+) T cell subpopulations distinctly control the prognosis of colorectal cancers. Nat. Med. 22, 679-684 (2016).

49. Thiele Orberg, E. et al. The myeloid immune signature of enterotoxigenic Bacteroides fragilis-induced murine colon tumorigenesis. Mucosal Immunol. 10, 421-433 (2016).

50. Gur, C. et al. Binding of the Fap2 protein of Fusobacterium nucleatum to human inhibitory receptor TIGIT protects tumors from immune cell attack. Immunity 42, 344-355 (2015)

51. Castellarin, M. et al. Fusobacterium nucleatum infection is prevalent in human colorectal carcinoma. Genome Res. 22, 299-306 (2012).

52. Li, Y. Y. et al. Association of fusobacterium nucleatum infection with colorectal cancer in Chinese patients. World J. Gastroenterol. 22, 3227-3233 (2016).

53. Warren, R. L. et al. Co-occurrence of anaerobic bacteria in colorectal carcinomas. Microbiome 1, 16 (2013).

54. Kolenbrander, P. E., Andersen, R. N. \& Moore, L. V. Coaggregation of Fusobacterium nucleatum, Selenomonas flueggei, Selenomonas infelix, Selenomonas noxia, and Selenomonas sputigena with strains from 11 genera of oral bacteria. Infect. Immun. 57, 3194-3203 (1989).

55. Kolenbrander, P. E. \& London, J. Adhere today, here tomorrow: oral bacterial adherence. J. Bacteriol. 175, 3247-3252 (1993).

56. Johnson, $\mathrm{C}$. H. et al. Metabolism links bacterial biofilms and colon carcinogenesis. Cell Metab. 21, 891-897 (2015)

57. Flemer, B. et al. Tumour-associated and non-tumour-associated microbiota in colorectal cancer. Gut https://doi.org/10.1136/gutjnl-2015-309595 (2016).

58. Harris, P. A. et al. Research electronic data capture (REDCap)--a metadata-driven methodology and workflow process for providing translational research informatics support. J. Biomed. Inform. 42, 377-381 (2009).
59. Fadrosh, D. W. et al. An improved dual-indexing approach for multiplexed $16 \mathrm{~S}$ rRNA gene sequencing on the Illumina MiSeq platform. Microbiome 2, 6 (2014).

60. Caporaso, J. G. et al. Ultra-high-throughput microbial community analysis on the Illumina HiSeq and MiSeq platforms. ISME J. 6, 1621-1624 (2012).

61. Klindworth, A. et al. Evaluation of general $16 \mathrm{~S}$ ribosomal RNA gene PCR primers for classical and next-generation sequencing-based diversity studies. Nucleic Acids Res. 41, e1 (2013).

62. Bartram, A. K., Lynch, M. D., Stearns, J. C., Moreno-Hagelsieb, G. \& Neufeld, J. D. Generation of multimillion-sequence 16S rRNA gene libraries from complex microbial communities by assembling paired-end illumina reads. Appl. Environ. Microbiol. 77, 3846-3852 (2011).

63. Magoc, T. \& Salzberg, S. L. FLASH: fast length adjustment of short reads to improve genome assemblies. Bioinformatics 27, 2957-2963 (2011).

64. Bolger, A. M., Lohse, M. \& Usadel, B. Trimmomatic: a flexible trimmer for Illumina sequence data. Bioinformatics 30, 2114-2120 (2014).

65. Kuczynski, J. et al. Using QIIME to analyze 16S rRNA gene sequences from microbial communities. Curr. Protoc. Bioinformatics 10, 1-20 (2011).

66. Caporaso, J. G. et al. PyNAST: a flexible tool for aligning sequences to a template alignment. Bioinformatics 26, 266-267 (2010).

67. Bragg, L., Stone, G., Imelfort, M., Hugenholtz, P. \& Tyson, G. W. Fast, accurate errorcorrection of amplicon pyrosequences using Acacia. Nat. Methods 9, 425-426 (2012).

68. Edgar, R. C., Haas, B. J., Clemente, J. C., Quince, C. \& Knight, R. UCHIME improves sensitivity and speed of chimera detection. Bioinformatics 27, 2194-200 (2011).

69. Langmead, B. \& Salzberg, S. L. Fast gapped-read alignment with Bowtie 2. Nat. Methods 9, 357-359 (2012).

70. McDonald, D. et al. An improved Greengenes taxonomy with explicit ranks for ecological and evolutionary analyses of bacteria and archaea. ISME J. 6, 610-618 (2012).

71. Wang, Q., Garrity, G. M., Tiedje, J. M. \& Cole, J. R. Naive Bayesian classifier for rapid assignment of rRNA sequences into the new bacterial taxonomy. Appl. Environ. Microbiol. 73, 5261-5267 (2007).

72. Langille, M. G. et al. Predictive functional profiling of microbial communities using $16 \mathrm{~S}$ rRNA marker gene sequences. Nat. Biotechnol. 31, 814-821 (2013).

Open Access This article is licensed under a Creative Commons Attribution 4.0 International License, which permits use, sharing, adaptation, distribution and reproduction in any medium or format, as long as you give appropriate credit to the original author(s) and the source, provide a link to the Creative Commons license, and indicate if changes were made. The images or other third party material in this article are included in the article's Creative Commons license, unless indicated otherwise in a credit line to the material. If material is not included in the article's Creative Commons license and your intended use is not permitted by statutory regulation or exceeds the permitted use, you will need to obtain permission directly from the copyright holder. To view a copy of this license, visit http://creativecommons. org/licenses/by/4.0/.

(c) The Author(s) 2017 